\title{
La Física de los Viajes en el Tiempo a través de un Agujero de Gusano
}

\author{
RAÚL ISEA ${ }^{1}$ \\ ${ }^{1}$ Fundacion Instituto de Estudios Avanzados-IDEA, Hoyo de la Puerta, Venezuela, mail: raul.isea@gmail.com
}

Recibido: 1 de Febrero de 2016 / Aceptado: 1 de Mayo de 2016

\begin{abstract}
Resumen
This paper introduces us into the physics of time travel employing wormholes. It is highlighted how cinematography plunges us into possible temporal paradoxes or contradictions, but that we can reinterpret under the Novikov principle of self-consistency, explaining that the only possible events are the ones that don't change the future. On the other hand, a mental exercise is developed with a billiard ball that travels to the past through a wormhole, to collide with its earlier itself, like the grandfather paradox. In addition, wormholes are analyzed from the point of view of General Relativity, and how they link to Einstein's Field of Theory.
\end{abstract}

Keywords: Time, Einstein, Wormhole, Relativity, Field Theory

\begin{abstract}
El presente trabajo nos introduce en la física de los viajes en el tiempo empleando para ello los agujeros de gusano. Se resalta cómo la cinematografía nos sumerge en posibles paradojas o contradicciones temporales, pero que se pueden reinterpretar bajo el principio de autoconsistencia de Nóvikov, que explica que son solo posibles aquellos eventos que no logran cambiar el futuro. Por otra parte, se desarrolla un ejercicio mental con una bola de billar que viaja al pasado cuando atraviesa un agujero de gusano para que posteriormente colisione consigo misma, claro símil de la paradoja de los abuelos. Asi mismo, se analizan los agujeros de gusano desde la óptica de la relatividad general, y cómo se vinculan con la teoría de campo de Einstein.
\end{abstract}

Palabras clave: Tiempo; Einstein; Agujero de gusano; Relatividad; Teoría de campo

\section{UNA INTRODUCCIÓN DE PELÍCULA}

G RaCias al cine, los viajes en el tiempo son un tema que cautiva al desafiar nuestra compresión de la realidad a través de paradojas temporales que destacan cómo algunos eventos no siempre son consecuencia de las causas que los originaron. Para comprenderlo, abordaremos tres películas de ciencia ficción: Contact, The Time Machine y Terminator.

Recordemos que la película Contact (Contacto) está basada en la novela escrita por Carl Edward Sagan (19341996) en 1985, y dirigida por Robert Zemeckis una década después. La protagonista, encarnada por Jodie Foster, es la doctora Eleanor Ellie Arroway, quien descubre que existe vida extraterrestre cuando logra descifrar una señal alienígena que daba instrucciones para construir una máquina capaz de viajar hasta donde vivían los autores del mensaje. Ellie finalmente concreta el viaje atravesando varios agujeros de gusano (también conocidos en la literatura científica como puentes de Einstein-Rosen), hasta que consigue conversar con un extraterrestre que toma la forma de su padre fallecido. $\mathrm{Al}$ regresar al complejo de lanzamiento, la doctora Arroway se percata de que su aventura duró aproximadamente 18 horas, pero para los científicos ubicados en la sede de Hokkaido, en Japón, donde está la plataforma de lanzamiento, el viaje fue de unos pocos segundos. Hacia el final de la película, en una conversación entre el asesor de seguridad Michael Kitz y la jefa de Gabinete de la Casa Blanca (Rachel Constantine), admiten efectivamente que la cámara de grabación de la doctora Arroway grabó dieciocho horas de pura estática. En este punto nos preguntamos: ¿realmente es posible explicar este desfase temporal experimentado por la doctora Ellie?

El siguiente largometraje es el remake de The Time Machine (La máquina del tiempo). Una de las primeras películas sobre los viajes del tiempo fue justamente la versión del mismo nombre, de 1960, dirigida y producida por George Pal, basada en la novela de 1895 escrita por H. G. Wells. La nueva versión fue estrenada en 2002, y fue dirigida por Simon Wells, biznieto del escritor. Guy Pearce interpretó al protagonista, un científico que residía en Nueva York hacia 1899 llamado Alexander Hartdegen. Una noche cuando caminaba junto a su novia Emma (interpretada por Sienna Guillory), él decide comprometerse con ella, pero de pronto aparece un ladrón que los asalta y la asesina. Alexander se obsesiona con viajar al pasado para impedir ese hecho, pero pese a sus intentos de evitarlo, la señorita Emma siempre moría, razón por la cual decide viajar al futuro para averiguar por qué no logra cambiar el pasado. Así, llega a un lejano año 802.701, en el que 
Alexander descubre que hay dos civilizaciones distintas: los Eloi y los Morlocks, separados tras el colapso de la explosión de la luna. Al final de la película, el líder que controla a los Morlocks le explica que no puede cambiar el destino de Emma porque su muerte fue lo que le impulsó a construir la máquina del tiempo. Si ella hubiera vivido, nunca la hubiera construido.

En la película The Terminator, dirigida por James Cameron en 1984, la protagonista Sarah Connor, interpretada por Linda Hamilton, tiene un hijo que será el líder de la resistencia contra los robots en el futuro. Para evitar que él naciera, las máquinas deciden enviar a un terminator, el modelo $T-800$, interpretado por Arnold Schwarzenegger, con el encargo de asesinarla, y así frenar de raíz la rebelión en su época. Al enterarse, la resistencia decide a enviar a Kyle Reese (el actor Michael Biehn) para rescatar a Sarah e impedir que el terminator cumpla su misión. Curiosamente, el soldado que proviene del futuro para salvarla en el pasado, será el padre del líder de la revolución John Connor, quien decidió mandarlo al pasado para proteger a su mamá. Surge entonces la pregunta: ¿quién fue el padre de John antes de que viniera del futuro Kyle?

Estos ejemplos nos ilustran cómo las acciones del futuro parecen estar condicionando el pasado, lo que probablemente conduce a cuestionar la validez de los viajes en el tiempo, en vista de que en nuestra realidad física, sentimos que todo evento es consecuencia de una causa. Sin embargo, y como veremos más adelante, será posible mostrar que probablemente no existen estas paradojas temporales.

\section{II. ¿PODEMOS EXPLICAR ESTAS "PARADOJAS TEMPORALES"?}

Comencemos con la paradoja de los abuelos. Esta fue acuñada por el francés René Barjavel (1911 - 1985) en su novela Le voyageur imprudent (El viajero imprudente) publicada en 1944, y traducida al inglés en 1958. En ella se enuncia que si una persona viaja al pasado y mata a su abuelo, dicha acción evitará que nazca su padre, de modo que si el abuelo no vive, entonces es imposible que el viajero pudiera haber nacido para luego asesinarlo.

En el contexto de la tercera película mencionada más arriba, la inteligencia artificial que dominaba a los robots (llamada Skynet) envió al terminator desde 2029 a 1984 para asesinar a Sarah Connor, y así evitar que naciera John. Sin embargo, como se comentó con la película The Time Machine, vislumbramos una clara solución a esta aparente contradicción, ya que sería imposible que el terminator eliminara a Sarah porque al hacerlo, ella nunca hubiera concebido a John y, por ende, el terminator nunca hubiera sido enviado al pasado para asesinarla.

En esto último más bien parece que estuviésemos avecinándonos a una paradoja de la predestinación, es decir, el viajero del tiempo cumple un papel protagónico en los hechos que ocurren en el pasado como se destacaron en cada una de las películas, y sin su acción o intervención, no existiría el futuro plasmado en ellas. De modo que estamos concluyendo, cualitativamente hablando, que las acciones no pueden cambiar el futuro porque ya todo está predestinado, y en cierta forma, pareciera que estuvieran atrapadas en un ciclo o también llamado bucle temporal.

En este punto es necesario citar el principio de auto consistencia de Nóvikov [1, conocido también como la conjetura de consistencia de Nóvikov (desarrollada en los años ochenta). Este astrofísico resolvió el problema de la paradoja de los abuelos indicando que si un evento modifica el pasado, entonces la probabilidad de que este ocurra es cero, es decir, nula; lo que conduce indudablemente a que no existirán paradojas. En el contexto de las películas, Skynet logra viajar al pasado porque esa acción no modificará el futuro que ellos desean alterar con la muerte de Sarah Connor. Por ello, ahora somos capaces de comprender por qué Alexander no logra evitar que muera Emma a pesar de sus intentos en retroceder al pasado.

Seguidamente, discutiremos la paradoja de Polchinski, que se puede reinterpretar como la paradoja de los abuelos, y mostraremos cómo el presente y el pasado interactúan de forma conjunta, empleando aspectos básicos de la física clásica y la geometría. Pudiera considerarse como un ejemplo banal, pero el año pasado fue publicado en la revista Nature un trabajo sobre cómo un fotón viaja al pasado para colisionar consigo mismo [2].

\section{Auto COLISión DE UNA BOLA DE BILLAR}

El siguiente ejercicio mental fue planteado y desarrollado por Dolansky y Krtous [3]. Imaginemos por un momento una mesa de billar en la que dos de sus agujeros son en realidad las bocas de entrada y salida de un agujero de gusano (véase la figura 1). El agujero se caracteriza por el hecho de que una bola de billar ingresa por uno de sus extremos, supongamos que entra por la boca derecha señalada con la letra B, pero emerge por el extremo izquierdo (indicado con la letra A) un tiempo atrás, de manera tal que dicha bola de billar viaja al pasado y al salir colisiona consigo misma. El análisis de este ejemplo puede ser la base para comprender el problema que resolvieron experimentalmente con el fotón.

Si somos críticos, y consideramos que la bola de billar viajó al pasado, debemos ser capaces de deducir que probablemente la bola de billar salió del agujero de gusano antes de ingresar en él. En tal sentido, Joseph Polchinski formula la siguiente paradoja, conocida en la literatura científica como la paradoja de Polchinski: si la bola que sale del agujero de gusano proveniente del pasado sale antes de que pueda entrar al mismo, entonces ¿de dónde provino la bola del pasado para que pueda colisionar consigo misma? (véase la figura 1). Es un claro símil de la paradoja de los abuelos. 


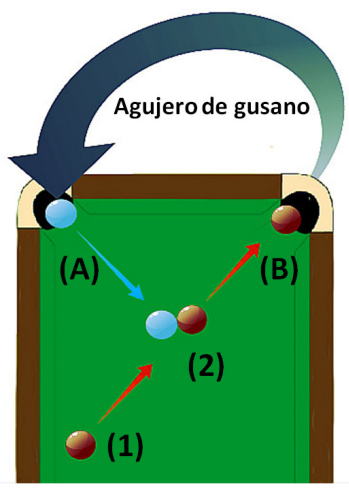

Figura 1: Trayectoria de una bola de billar que viaja a través de un agujero de gusano (señalado este último con una flecha degradada en color azul oscuro en la parte superior de la figura) retrocediendo la bola en el tiempo para que pueda colisionar consigo misma al llegar al punto 2. En color rojo, se visualiza la bola que inicialmente proviene de algún punto de la mesa de billar atravesando la posición 1, con dirección a la entrada del agujero de gusano señalado con la letra B. Posteriormente, viaja al pasado hasta que sale del agujero en el punto $A$ de color azul claro, hasta colisionar consigo misma en el punto 2 .

Sin embargo, cuando la bola de billar atraviesa el túnel de gusano, desde su perspectiva ha transcurrido un tiempo propio y a su salida del agujero, choca con lo que a ella le parece 'otra' bola que está en su trayectoria. De hecho, y en analogía con la película Contact, fue justamente lo que le ocurrió a la doctora Ellie cuando viajó por el agujero de gusano por más de dieciocho horas. En esa cinta se observa que hay dos historias diferentes: una desde el punto de vista de la protagonista que viaja en el tiempo, y otra con respecto al del observador externo, es decir, aquellos que estaban en Hokkaido, pero con la peculiaridad de que tanto los eventos iniciales (antes del viaje por el agujero de gusano) como los finales (cuando retornó a Hokkaido) coinciden en el tiempo.

Puede decirse que las películas de ciencia ficción no proponen paradojas temporales sino que efectivamente somos capaces de explicarlas a partir de las leyes del mundo real.

\section{ECUACIÓN DE MOVIMIENTO}

A continuación se demostrará que realmente no existe una paradoja temporal con el ejemplo de la auto colisión de la bola de billar. Para ello, es necesario plantear las siguientes suposiciones. Los extremos del agujero de gusano están en reposo con respecto al marco inercial, y lo restringiremos en un plano bidimensional, no esférico, con una línea límite común que llamaremos eje, formando así dos medios-planos paralelos de los extremos del agujero de gusano, separados entre sí por un ángulo $\gamma$ (véase la figura 27. Consideremos, asimismo, el rango de valores de $\gamma$ entre $(\pi / 2, \pi)$. Supongamos además que el movimiento

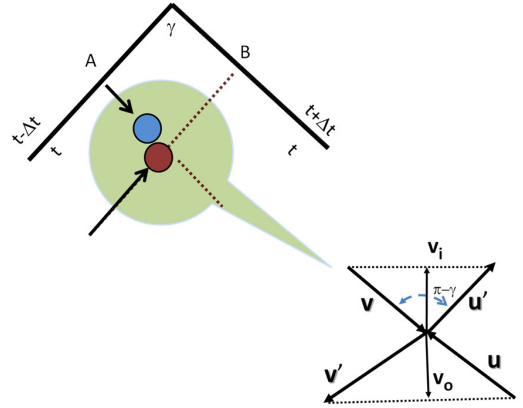

Figura 2: Reducción esquemática de la auto colisión de una bola de billar en el tiempo $t$ que proviene de alguna parte de la mesa de billar (representada con color rojo en la figura anterior), y alcanza el agujero de gusano en el extremo indicado con la letra $B$, hasta que sale por el otro lado en el punto A. Dicho viaje transcurrió en un intervalo de tiempo $\Delta t$. En la parte inferior izquierda se esquematiza justamente el punto de la auto colisión, como se explicará en el texto.

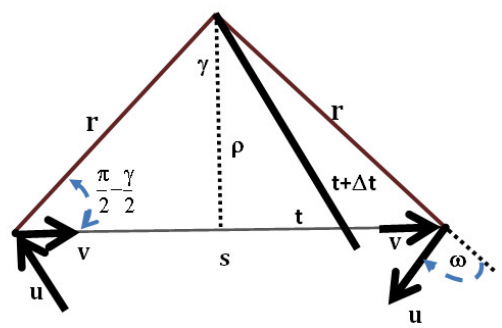

Figura 3: Detalles de la geometría del problema (véase explicación en el texto).

de la bola de billar es no-relativista, y que el radio de la bola es despreciable (es decir, $R \rightarrow 0$ ), así como que la velocidad de la bola no cambia cuando atraviesa el agujero ni cuando colisiona.

Hemos indicado que la bola posee una dirección que va hacia el punto $B$ y al viajar al pasado colisiona consigo misma (emerge en el punto $A$ ), por lo que es necesario determinar el parámetro de impacto, definido como $\rho$, que nos indicará la distancia inicial necesaria para que ocurra la colisión. Así mismo, es necesario definir una distancia que llamaremos radial, denotada con la letra $r$, la cual será aquella que indica la distancia desde el punto donde ocurre la auto colisión hasta el extremo del agujero de gusano. Gracias a esta última variable, $r$, es posible definir el ángulo $\omega$ dado por la dirección de la trayectoria de la partícula que sale del agujero de gusano (la que viajó al pasado) y es posible describir las condiciones en la trayectoria de la bola para que ocurra esa auto colisión.

Si además definimos la variable $s$ como se aprecia en la figura 3 que es resultado de la distancia que va desde los extremos del agujero de gusano, se desprende que $\rho=r \cdot \operatorname{sen}(\omega)$, y además se cumple que $\rho=r \cdot \cos (\gamma / 2)$, por lo que al despejar el valor de $r$ anterior, tendremos:

$$
r=\frac{\rho}{\cos (\gamma / 2)}
$$


Si empleamos la ecuación 1 y tenemos presente la definición de la tangente, concluimos que el valor de $s$ será:

$$
s=2 \rho \cdot \tan \left(\frac{\gamma}{2}\right)=2 r \cdot \operatorname{sen}\left(\frac{\gamma}{2}\right)
$$

Al determinar la velocidad de la bola de billar $(v>0)$ mientras está recorriendo la distancia $s$ en el intervalo de tiempo $\Delta t$, obtenemos:

$$
v=\frac{s}{\Delta t}=\frac{2 r \cdot \operatorname{sen}\left(\frac{\gamma}{2}\right)}{\Delta t}
$$

En vista de que hemos supuesto que la velocidad de la bola es la misma sin cambiar por efecto de la colisión ni cuando atraviesa el agujero de gusano, por conservación del momento angular entre la auto colisión, $v \cdot \operatorname{sen}(\gamma / 2)$ debe ser igual al momento angular de la trayectoria de entrada al agujero dado por $u \cdot \cos (\omega)$, es decir, $v \cdot \operatorname{sen}(\gamma / 2)=u \cdot \cos (\omega)$ por lo que podemos despejar el valor de $v$, que será igual a

$$
v=\frac{u \cdot \cos (\omega)}{\operatorname{sen}(\gamma / 2)}
$$

De modo que al igualar las ecuaciones 3 y 4 , podemos despejar el valor de la distancia radial $r$, es decir:

$$
r=\frac{u \Delta t \cdot \cos (\omega)}{2 \operatorname{sen}^{2}(\gamma / 2)}
$$

Si tenemos presente que $\rho=r \cdot \operatorname{sen}(\omega)$ como se explicó anteriormente, y teniendo en cuenta la ecuación 5 . el valor de $\rho$ será igual a

$$
\begin{aligned}
\rho=r \cdot \operatorname{sen}(\omega) & =\frac{u \Delta t \cdot \cos (\omega) \operatorname{sen}(\omega)}{2 \operatorname{sen}^{2}(\gamma / 2)}= \\
& =\frac{u \Delta t \cdot \operatorname{sen}(2 \omega) \csc ^{2}(\gamma / 2)}{4}
\end{aligned}
$$

En la figura 4 se observa que el valor máximo del parámetro ocurre cuando $\omega=\pi / 4$. A partir del mismo, es posible determinar la mayor magnitud del parámetro de impacto $\left(\rho_{\max }\right)$ dado por la ecuación 6 , donde $\cos (\pi / 4)$. $\operatorname{sen}(\pi / 4)$ es igual a $1 / 2, \mathrm{y}$ se obtiene

$$
\rho_{\max }=\frac{u \Delta t}{4 \operatorname{sen}^{2}(\gamma / 2)}
$$

Por lo que advertimos que la máxima amplitud del parámetro de impacto depende de la velocidad de la bola de billar, el tiempo que tarda en atravesar el agujero, así como el ángulo en que están dispuestos los extremos del agujero de gusano (ecuación 7). Más aún, la figura 4 nos está indicando que el parámetro de impacto oscila, presentando valores positivos (los que reflejan una colisión en el pasado), mientras que los valores negativos son el resultado de una auto colisión en el futuro.

Tenemos entonces las expresiones matemáticas de la distancia radial $r$ y el parámetro de impacto $\rho$ en función de las variables $u, \Delta t$ y $\rho$. Solo resta expresar cómo deben

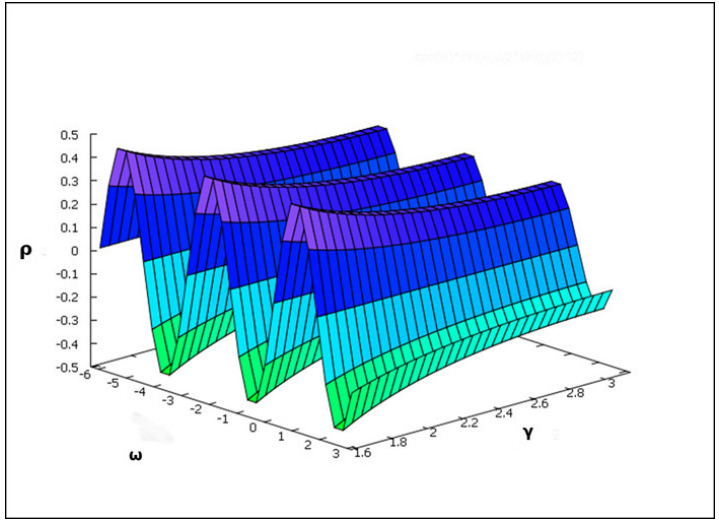

Figura 4: Gráfico 3D del parámetro de impacto $\rho$ calculado de acuerdo a la ecuación 6 en función de $\omega$ entre $(-2 \pi, \pi)$, mientras que $\gamma$ está comprendido entre $(\pi / 2, \pi)$.

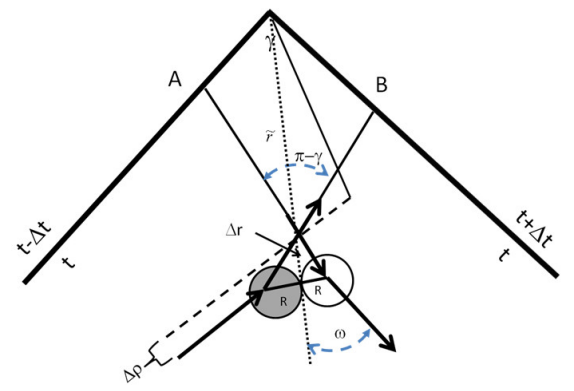

Figura 5: Modelo realista de la auto colisión de una bola de billar de radio $R$.

variar los ángulos $\omega$ y $\gamma$ para que ocurra la auto colisión. Esta se deduce fácilmente según muestra la figura 5 Estará dada por

$$
\omega=\frac{\pi-\gamma}{2}
$$

De modo que al sustituir el valor de $\omega$ dado por la ecuación 8 en la ecuación 6 , obtenemos

$$
\rho=\frac{u \Delta t \cdot \csc ^{2}(\gamma / 2) \operatorname{sen}(\gamma)}{4}
$$

En cambio, si se hubiera sustituido $\gamma / 2$ como $(\pi / 2-$ $\omega)$ de acuerdo a la ecuación 8 , entonces la expresión de $\rho$ sería

$$
\rho=\frac{u \Delta t \cdot \tan (\omega)}{2}
$$

de manera que, efectivamente, el parámetro de impacto está dado por el ángulo $\gamma$, la magnitud de la velocidad inicial y el tiempo que transcurre durante el viaje en el tiempo $(\Delta t)$, como se describió en la ecuación 7

\section{Construyendo un modelo más REALISTA}

Proponemos ahora una bola de billar con un radio determinado $(R)$ y distinto de cero, pero cuya trayectoria aún sigue siendo no-relativista (como se ilustra en la 
figura 5. La distancia radial $r$ será igual a la distancia medida por la auto intersección de la trayectoria interna (denotada como $\tilde{r}$ ) más una diferencia producto de los radios de las bolas de billar dada por $\Delta r$; de modo que $r=\tilde{r}+\Delta r$.

Determinaremos el parámetro de impacto, pero primero tenemos que considerar la diferencia de la distancia radial debido al radio de la bola. Análogamente a la bola de billar sin radio, el parámetro de impacto (ahora definido como $\tilde{\rho}$ ) será función de la distancia $\tilde{r}$, por lo que $\tilde{\rho}=\tilde{r} \operatorname{sen}(\omega)$. Lógicamente, será de esperar que la distancia $s$ se modificará para considerar las pequeñas diferencias relativas a los radios de la bola de billar, como se muestra en la figura 5 De hecho, somos capaces de discernir que $\Delta s=R / \cos (\gamma / 2)$, mientras que $\tilde{s}=2 \tilde{r} \operatorname{sen}(\gamma 2)$. Por ello el valor de $s$ será simplemente

$$
s=\tilde{s}+2 \Delta s=2 \tilde{r} \operatorname{sen}(\gamma / 2)+\frac{2 R}{\cos (\gamma / 2)}
$$

Entonces la velocidad de la bola será justamente el valor de $s$ entre $\Delta t$, es decir:

$$
v=\frac{2}{\Delta t}\left[\tilde{r} \operatorname{sen}(\gamma / 2)+\frac{R}{\cos (\gamma / 2)}\right]
$$

Aún se sigue cumpliendo la relación deducida en la ecuación 4 ya que las proyecciones de la velocidad son las mismas, de modo que se mantiene la relación $u \cdot \cos (\omega)=$ $v \cdot \operatorname{sen}(\gamma / 2)$, que al sustituir por la ecuación anterior, nos da en este caso:

$$
\begin{array}{r}
u \cdot \cos (\omega)=\left\{\frac{2}{\Delta t}\left[\tilde{r} \operatorname{sen}(\gamma / 2)+\frac{R}{\cos (\gamma / 2)}\right]\right\} \times \\
\times \operatorname{sen}(\gamma / 2)
\end{array}
$$

De esta última relación podemos despejar el valor de $\tilde{r}$, es decir:

$$
\begin{array}{r}
\tilde{r}=\csc (\gamma / 2) \times \\
\times\left[\frac{u \Delta t \cdot \cos (\omega) \csc (\gamma 2)}{2}-R \cdot \sec (\gamma / 2)\right]
\end{array}
$$

Al sustituir la expresión de $\tilde{r}$ en el parámetro de impacto $\tilde{\rho}=\tilde{r} \operatorname{sen}(\omega)$, y haciendo un poco de álgebra para no saturar con tantas ecuaciones este trabajo, conseguimos:

$$
\begin{array}{r}
\tilde{\rho}=\left[\csc (\gamma / 2) \cdot \frac{u \Delta t \cdot \operatorname{sen}(2 \omega) \csc (\gamma 2)}{4}\right]- \\
-[\csc (\gamma / 2) \cdot R \cdot \operatorname{sen}(\omega) \cdot \sec (\gamma / 2)]
\end{array}
$$

Si hacemos $R \rightarrow 0$ en la última ecuación, que sería el caso de una bola de billar puntual como el deducido en la primera parte de este trabajo, logramos exactamente la misma ecuación 6, de manera que el segundo término es consecuencia de considerar un radio finito en la bola de billar.

Como se ha indicado hasta el momento, hemos sido capaces de mostrar matemáticamente que es posible que una bola de billar viaje al pasado, al punto en que colisiona consigo misma sin introducir ninguna suposición referente al funcionamiento del agujero de gusano responsable del viaje al pasado.

\section{Conjetura de Protección de la CRONOLOGÍA}

En este punto, no podemos pasar por alto la conjetura de protección de la cronología propuesta por Stephen Hawking [4], la cual sostiene que es imposible viajar en el tiempo a escala macroscópica, y por ende, no tenemos contradicciones en el tiempo propiamente dichas. Hawking mostró que al introducir la relatividad general en la formulación de la mecánica cuántica, las fluctuaciones de la energía del vacío incrementan la densidad de energía al infinito justo en el momento en el que se va a viajar al pasado, lo cual termina por destruir la máquina del tiempo en el mismo instante en que ha sido creada. En otras palabras: el Universo, a través de las leyes de la física, impide cambiar el pasado.

Sin embargo, recientemente se observó experimentalmente que la conjetura propuesta por Hawking se debe reexaminar con mayor atención. Martin Ringbauer y colaboradores, de la Universidad de Queensland, en Australia, diseñaron un experimento con equipos ópticos que muestra cómo un fotón de luz viaja al pasado hasta que interactúa consigo mismo gracias a que emplea atajos en el espacio-tiempo, simulando el pasaje a través de un agujero de gusano [2]. A raíz de este experimento, se pudiera descartar la validez de la conjetura de protección de la cronología de Hawking. Sin embargo, es necesario desarrollar una teoría cuántica de la gravedad para poder corroborar esta última afirmación.

Una vez que hemos destacado que viajar en el tiempo es un hecho posible desde una perspectiva clásica y norelativista, solo resta entender esa 'caja negra' que hemos llamado agujero de gusano. Dicho estudio lo haremos en dos partes. Primero se determinará qué conocemos y cómo se caracterizan los agujeros de gusano, y finalmente adelantaremos algunos detalles del estudio de los agujeros de gusano para comprender la teoría de la relatividad general. Este último tema se desarrollará en un próximo trabajo, pero no por ello se debe pasar por alto el hecho de que los agujeros de gusano son el puente para comprender el espacio-tiempo que gobierna la cosmología.

\section{Agujero de gusano}

Gracias a los estudios realizados por Albert Einstein (1879 - 1955) y Nathan Rosen $(1909$ - 1995) en 1935, publicados en un trabajo científico cuyo título traducido sería El problema de la partícula en la teoría general de la relatividad [5], propusieron un modelo geométrico de una partícula de física elemental donde el espacio estaba representado por dos hojas idénticas paralelas entre sí, tal que la partícula sería un puente que las conecta evitando 
así las singularidades. De allí el nombre de puente de Einstein-Rosen (en el apéndice A se muestra el resultado, grosso modo, de la deducción).

El nombre de agujero de gusano (traducción de la palabra inglesa wormhole) fue introducido por John A. Wheeler [6], pero desde ese entonces, el problema es que nunca se han observado en el universo, sino que más bien han sido blanco de estudios teóricos que han brindado la posibilidad de viajar grandes distancias en el espacio en el menor tiempo posible, así como también ser la base para realizar posibles viajes en el tiempo (mayores detalles en los libros [7-9]).

Actualmente se plantea que de existir un agujero de gusano, sería por apenas un instante, y que tal acción requeriría grandes cantidades de energía negativa, en vista de que una energía positiva (como ya sabemos) atrae a la materia y lo colapsaría, mientras que una energía negativa genera el efecto contrario que es justamente la esencia del agujero de gusano que nos permite el paso de materia a través de él.

Algunos teóricos han señalado que esta energía negativa sería precisamente la de Casimir [10]. Hacia 1948 se estableció que en el vacío, al colocar dos placas paralelas y completamente lisas lo más juntas posible, se puede atrapar la energía por los efectos cuánticos asociados al vacío, los cuales han sido medidos experimentalmente al atraerse esas placas. No se debe subestimar este tipo de energía porque es el tema de investigación en la NASA para los viajes espaciales 11 al ser la esencia de los propulsores-Q (Q-thruster). Dicha implementación está lejos de ser real en vista de que el impulso obtenido gracias al efecto Casimir es muy débil en comparación con el requerido para mover una nave espacial. Se trataría más bien de destacar que el vacío realmente no está vacío sino que alberga una serie de partículas virtuales protagonistas y causantes del efecto Casimir antes señalado.

Esta última idea no debe descartarse, como lo ha indicado Butcher [10, quien demostró matemáticamente que si la garganta del agujero de gusano (representada con la variable $a$ de la figura 6) es muy extensa $(a>r)$ en comparación con la boca del agujero (medido a través de la variable $r$ ), este generará energía negativa en su centro, que es justamente la necesaria para que pudiera existir, aunque lamentablemente sería por un breve instante, condición necesaria para generar la apertura del túnel, y muy estrecha, lo cual permitiría solo el paso de fotones (actualmente se está preparando un ensayo que explica este formalismo físico matemático para incentivar futuros trabajos).

Llegados a este punto, no podemos dejar de mencionar la película Interstellar (dirigida por Christopher Nolan) en la que se nos muestra el viaje de una nave espacial a través de un agujero de gusano, mediante imágenes obtenidas gracias a estudios cosmológicos derivados por Morris y Thorne [12]. Si disfrutan de la película estrenada en 2014, podrán observar que en el momento anterior a que la nave atraviesa el agujero, se pueden observar galaxias que están en el otro extremo de acuerdo a las

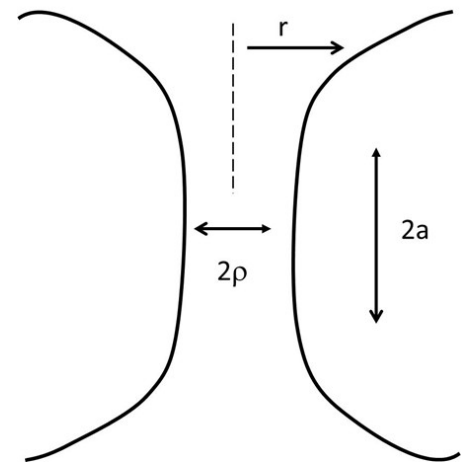

Figura 6: Esquema de un agujero de gusano.

leyes de la física, y que esa travesía no fue inmediata.

Permítanme terminar describiendo la anatomía de los agujeros de gusano, y cómo ellos están íntimamente relacionados con las ecuaciones de campo de Einstein, que posibilitan describir la gravedad como una distorsión del espacio-tiempo.

\section{FORMALISMO MATEMÁtiCO DEL AGUJERO DE GUSANO}

En el apéndice $\mathrm{B}$ se introduce rápidamente la métrica requerida en la Relatividad General que es la base para describir la topología del agujero de gusano dado por la siguiente expresión del elemento de línea $\left(d s^{2}\right)$ que nos indica la distancia espacio-temporal entre dos eventos, descrita en coordenadas esféricas:

$$
d s^{2}=-d t^{2}+d l^{2}+r^{2}\left(d \theta^{2}+\operatorname{sen}^{2} \theta d \phi^{2}\right)
$$

En muchos estudios de los agujeros de gusano se emplea un valor de radio $r$ igual a $\sqrt{\rho^{2}+l^{2}}$, donde $\rho$ es constante (se ven en la figura 6). De esta última relación se observa que para $l$ tendiendo a cero, el radio es justamente $\rho$ que representa el radio de la garganta del agujero de gusano (véase la figura 6). En caso contrario, cuando $l$ es mucho mayor que $\rho$, el radio será $|l|$.

Wheeler conjuntamente con Robert Fuller [13] llegan a la conclusión de que el agujero de gusano basado en la métrica empleada por Einstein y Rosen es inestable, tal que se desintegra inmediatamente después de que se forma por lo que ni un fotón puede atravesarlo.

En 1988 se retoma en la literatura científica la posibilidad de que los agujeros pudieran servir de túneles para ser atravesados gracias al trabajo desarrollado por Kip Thorne y Mike Morris, denominándolo el agujero de gusano atravesable de Morris-Thorne. Posteriormente Matt Visser [14 mostró que es posible atravesar un agujero de gusano sin necesidad de atravesar una región de materia exótica en el Universo (es decir, energía negativa) expresándolo como Lorentzian Wormholes gracias a la interacción con las cuerdas cósmicas.

A raíz de la película Interstellar, se incorporó el efecto de la gravedad (como una interacción débil) en la ecuación 
$d s^{2}$, de manera que la geometría del agujo de gusano es simplemente [15]:

$$
d s^{2}=-(1+2 V) d t^{2}+d l^{2}+r^{2}\left(d \theta^{2}+\operatorname{sen}^{2} \theta d \phi^{2}\right)
$$

Considerando que la gravedad está dada por $g=$ $|d V / d l|$, es decir, el módulo del potencial gravitatorio $V$ en virtud de que el potencial es negativo. Fijando idea de las dimensiones, teniendo presente que una gravedad similar a la tierra de $10 \mathrm{~m} / \mathrm{s}^{2}$, con un radio $\rho$ igual a un kilómetro, de modo que el potencial es $|V|$ extremadamente pequeño, el cual no es suficiente para modificar el espacio-tiempo, haciendo imposible atravesarlo, porque el agujero de gusano es consecuencia de la deformación del espacio-tiempo gracias a valores muy altos de gravedad.

Thorne logra plasmar la imagen de su agujero de gusano para la película Interstellar, pero no crean que dichas ecuaciones son sencillas de implementar computacionalmente: fueron realizadas mediante la compañía de efectos visuales londinense Double Negative donde consideraron realmente efectos relativistas y algoritmos de Monte Carlo, ya que las imágenes mostradas en la cinta requirieron más de ochocientos Terabytes de datos y más de cien horas de cálculo.

\section{TeOría de CAmpo DE Einstein}

Las ecuaciones de campo de Einstein describen la interacción de la materia con la curvatura del espaciotiempo (ver por ejemplo Misner et al [16]; Carroll [17]) y por lo visto anteriormente, es la teoría idónea para describir las condiciones geométricas de los agujeros de gusano. En ese sentido, recordemos que esa teoría nos explica que la gravedad es función del tensor métrico que describe las propiedades geométricas del espacio-tiempo (esto es, distancia, ángulo, volumen), el cual está descrito por la siguiente ecuación de campo que se cumple para cada punto del espacio-tiempo:

$$
G_{\mu \vartheta}=\frac{8 \pi G}{c^{4}} T_{\mu \vartheta}
$$

donde $G_{\mu \vartheta}$ es el tensor de curvatura de Einstein, mientras que $T_{\mu \vartheta}$ es el tensor de energía-impulso, en el cual está implícita la cantidad de materia.

Recordemos que el tensor de curvatura se puede describir como

$$
G_{\mu \vartheta}=R_{\mu \vartheta}-\frac{1}{2} R g_{\mu \vartheta}+\Lambda g_{\mu \vartheta}
$$

donde $R_{\mu \vartheta}$ es el tensor de curvatura de Ricci, que es la traza del tensor de curvatura de Riemann, $R$ es el escalar de la curvatura de Ricci, y $\Lambda$ es la constante cosmológica.

Recordemos que el tensor de Ricci data de 1903 gracias a Gregorio Ricci-Curbastro (1853-1925), y se ha empleado para describir curvaturas en espacios menores a cuatro dimensiones, de modo que en el espacio-tiempo no describe completamente la curvatura. El mismo se determina a partir de los símbolos de Christoffel, es decir:

$$
R_{\mu \vartheta} \equiv \partial_{\lambda} \Gamma_{\mu \vartheta}^{\lambda}-\partial_{\vartheta} \Gamma_{\lambda \mu}^{\lambda}+\Gamma_{\lambda \sigma}^{\lambda} \Gamma_{\mu \vartheta}^{\sigma}-\Gamma_{\sigma \vartheta}^{\lambda} \Gamma_{\lambda \mu}^{\sigma}
$$

donde los símbolos de $\Gamma_{\mu \vartheta}^{\lambda}$ están definidos como:

$$
\Gamma_{\mu \vartheta}^{\lambda}=\frac{1}{2} g^{\lambda \sigma}\left(\partial_{\mu} g_{\vartheta \sigma}+\partial_{\theta} g_{\mu \sigma}-\partial_{\sigma} g_{\mu \theta}\right)
$$

Para comprender las distintas expresiones de las ecuaciones anteriores, determinemos las ecuaciones de campo de Einstein para un agujero de gusano de métrica $(2+1)$, que es la geometría más simple, y estará dado por la siguiente expresión del elemento de línea:

$$
d s^{2}=-d t^{2}+d r^{2}+\left(r^{2}+b^{2}\right) d \phi^{2}
$$

En este caso, el tensor métrico está dado por $g_{\alpha \beta}=$ $\operatorname{diag}\left[-1,1, r^{2}+b^{2}\right]$, por lo que $g^{\alpha \beta}=\operatorname{diag}\left[-1,1,1 /\left(r^{2}+\right.\right.$ $\left.\left.b^{2}\right)\right]$.

Los únicos símbolos de Christoffel $\Gamma_{\mu \vartheta}^{\lambda}$ distintos de cero son simplemente

$$
\Gamma_{r \phi}^{\phi}=\frac{r}{r^{2}+b^{2}} \cdot \Gamma_{\phi \phi}^{r}=-r
$$

Debemos determinar el tensor de Riemann y a partir de él, el tensor de Ricci desde la contracción del tensor de Riemann, cuya definición es

$$
R_{\sigma \mu \vartheta}^{\rho}=\partial_{\mu} \Gamma_{\mu \sigma}^{\rho}-\partial_{\vartheta} \Gamma_{\mu \sigma}^{\rho}+\Gamma_{\mu \lambda}^{\rho} \Gamma_{\mu \sigma}^{\lambda}-\Gamma_{\vartheta \lambda}^{\rho} \Gamma_{\mu \sigma}^{\lambda}
$$

En este caso, el único elemento distinto de cero del tensor de Riemann es $R_{\phi \phi r}^{r}$

$$
R_{\phi \phi r}^{r}=-\frac{b^{2}}{r^{2}+b^{2}}
$$

Por lo que los elementos diferentes de cero del tensor de Ricci son

$$
\begin{gathered}
R_{r r}=-\partial_{r} \Gamma_{r \phi}^{\phi}-\Gamma_{r \phi}^{\phi} \Gamma_{\phi r}^{\phi}= \\
=-\frac{b^{2}}{\left(r^{2}+b^{2}\right)^{2}}, R_{\phi \phi}=-\frac{b^{2}}{r^{2}+b^{2}}
\end{gathered}
$$

El valor de R, conocido como el escalar de la curvatura de Ricci, se puede determinar fácilmente a partir de la relación $R=g^{\mu \vartheta} R_{\mu \vartheta}$, por lo que obtenemos

$$
R=g^{r r} R_{r r}+g^{\phi \phi} R_{\phi \phi}=-\frac{2 b^{2}}{\left(r^{2}+b^{2}\right)^{2}}
$$

Sólo resta describir entonces el tensor energía-impulso (también conocido como tensor energía-momento) indicado en la ecuación 18 Como su nombre lo indica, describe el flujo de energía y momento de una distribución de materia. Si suponemos una constante cosmológica igual a cero, es decir, $\Lambda=0$, e igualamos las expresiones de las ecuaciones 18 y 19 , podemos obtener el tensor energía-impulso dado por

$$
T_{\mu \vartheta}=\left(\frac{8 \pi G}{c^{4}}\right)^{-1}\left[R_{\mu \vartheta}-\frac{1}{2} R g \mu \vartheta\right]
$$


$\mathrm{Al}$ evaluar con las cifras obtenidas anteriormente, el único elemento distinto de cero es

$$
\begin{aligned}
T_{00}=\left(\frac{8 \pi G}{c^{4}}\right)^{-1}[0 & \left.-\frac{1}{2}\left(-\frac{2 b^{2}}{\left(r^{2}+b^{2}\right)^{2}}\right)(-1)\right]= \\
= & -\left(\frac{8 \pi G}{c^{4}}\right)^{-1}\left[\frac{b^{2}}{\left(r^{2}+b^{2}\right)^{2}}\right]
\end{aligned}
$$

Se observa que es negativo el tensor por lo que se requiere una densidad de energía negativa para poder crear el agujero.

Resta por conocer qué propiedades se deben cumplir para que el agujero de gusano sea del tipo atravesable. En este punto Morris-Thorne [12] señalan:

1. La métrica debe ser simétrica y estática.

2. Las soluciones de las ecuaciones de campo de Einstein se debe cumplir en todo el espacio.

3. Se debe generar una garganta que conecta dos regiones asintótica mente planas del espacio-tiempo.

4. Debe carecer de un horizonte de evento.

5. Las fuerzas de marea gravitacional deben ser pequeñas.

6. El tensor de energía-momento generado por el agujero debe ser físicamente razonable.

7. Deben ser estables frente a perturbaciones del espaciotiempo.

La ecuación 16 se aprecia que cumple la primera condición (1.), al igual que la expresión 45, deducida en el apéndice B Esta última la podemos reescribir como:

$$
\begin{array}{r}
d s^{2}=-e^{2 \phi(r)} d t^{2}+ \\
+\frac{1}{1-\frac{b(r)}{r}} d r^{2}+r^{2} d \theta^{2}+r^{2} \operatorname{sen}^{2} \theta d \phi^{2}
\end{array}
$$

donde se ha sustituido las expresiones de $F(r)$ y $2 H(r)$ por $\phi(r)$ y $(1-b(r) / r)^{-1}$, respectivamente; de acuerdo a la expresión empleada por Morris y Thorne [12]. Dicha sustitución no es arbitraria porque las mismas determinan tanto la función del corrimiento al rojo (dado por la expresión $\phi(r))$, mientras que $b(r)$ condiciona la forma del agujero de gusano.

Para entender este último comentario, recordemos que el agujero de gusano posee simetría esférica, de modo que podemos considerar solo una rebanada espacial en un instante de tiempo fijo $t$ constante, de modo que la ecuación del elemento de línea será entonces obtenemos:

$$
d s^{2}=\frac{1}{1-\frac{b(r)}{r}} d r^{2}+r^{2} d \theta^{2}+r^{2} \operatorname{sen}^{2} \theta d \phi^{2}
$$

Por otra parte, consideramos una superficie euclidiana tridimensional cilíndrica con la misma geometría descrita por la siguiente ecuación:

$$
\begin{aligned}
d s^{2} & =d z^{2}+d r^{2}+r^{2} d \theta^{2}+r^{2} \operatorname{sen}^{2} \theta d \phi^{2}= \\
& =\left(1+\frac{d r^{2}}{d z^{2}}\right)+r^{2} d \theta^{2}+r^{2} \operatorname{sen}^{2} \theta d \phi^{2}
\end{aligned}
$$

Al comparar las ecuaciones del elemento de línea dadas por las ecuaciones 34 y 32 podemos inferir que:

$$
\left(1+\frac{d r^{2}}{d z^{2}}\right)=\frac{1}{1-\frac{b(r)}{r}}
$$

reordenando esta última expresión, obtenemos:

$$
\frac{d z}{d r}= \pm\left(\frac{r-b}{b}\right)^{-1 / 2}
$$

que es justamente una solución de una agujero de gusano caracterizado por el hecho que posee un radio mínimo de la garganta dado por $r=r_{0}=b(r)$.

Por otra parte, para satisfacer la condición (4.) y de esa manera se pueda viajar en ambos sentidos por el agujero de gusano, se debe garantizar que $g_{t t}=-e^{2 \phi} \rightarrow 0$, es decir, el intervalo de tiempo propio será nulo mientras el intervalo de tiempo sea finito.

Queda por comprender la diferencia de tiempo con que comenzamos este ensayo y así visualizar la factibilidad de los viajes en el tiempo considerando al agujero de gusano como una máquina del tiempo.

\section{Diferencia de tiempo COMO CONSECUENCIA DEL VIAJE DEL TIEMPO}

Ya somos capaces de explicar la diferencia de tiempo mostrada en la película Contact, y para ello observemos la figura 7. donde se muestra un agujero de gusano descrito por Morris, Thorne y Yurtsever, en el que se indican con los números 1 y 2 , dos eventos distintos caracterizados por el hecho de que el 1 está en reposo en comparación con el segundo, que está en movimiento (cercano a la velocidad de la luz).

De manera que la línea de suceso 1 sería la observada o presenciada en la sede de Hokkaido en Japón, mientras que la 2 correspondería al viaje de la doctora Ellie a través del agujero de gusano. Dicha figura destaca que ambos eventos comienzan y terminan en un mismo punto. De modo que lo que está ocurriendo es que el viaje de Ellie divide el espacio-tiempo en dos regiones distintas, y que esa superficie es lo que se denomina horizonte cronológico. Por ello, somos capaces de asociar ahora que la estabilidad del agujero de gusano es exactamente lo que va a condicionar la estabilidad del horizonte cronológico, a raíz de que el aumento de las fluctuaciones del vacío no permitiría la formación de ese agujero. Es importante no confundirlo con el horizonte de eventos que corresponde al límite de la superficie que delimita la región donde nada puede escapar. 


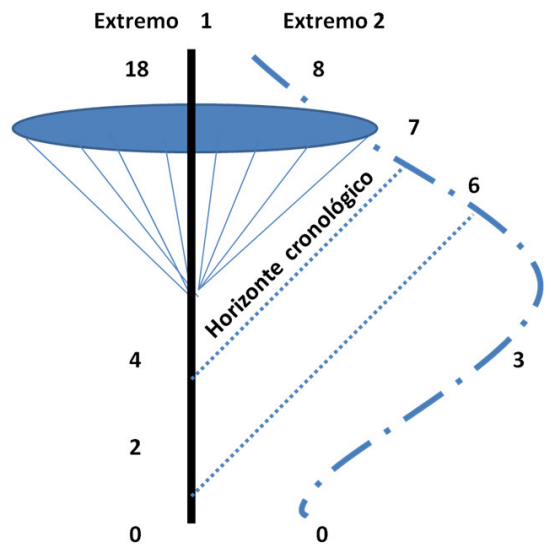

Figura 7: Esquema explicativo para comprender la diferencial temporal cuando un evento atraviesa un agujero de gusano que parte y llega al mismo punto.

\section{UNA POSIBLE EVIDENCIA EXPERIMENTAL}

Hasta la fecha no se ha podido observar un agujero de gusano, pero recientemente los trabajos de David Smith y sus colaboradores han permitido diseñar un dispositivo con la peculiaridad de que pueden recrear un agujero de gusano empleando la analogía con un túnel electromagnético, tal que un objeto atraviesa y reaparece en el extremo opuesto, gracias a la utilización de la radiación de microondas [18.

En su artículo publicado por Greenleaf y colaboradores [19], ellos se basan en las ecuaciones de Maxwell y son capaces de cambiar la topología del espacio frente a la presencia de ondas electromagnéticas. Este tipo de materiales los han denominado metamateriales, ya que son capaces de reflectar la luz en sus proximidades por lo que permiten hacerlo invisible a medida que lo atraviesa. De modo que los metamateriales nos brindan una posibilidad experimental de estudiar los agujeros de gusanos en nuestros laboratorios en vez de desde una pantalla de un computador.

\section{Conclusiones}

A lo largo del presente ensayo hemos destacado que la cinematografía nos ha brindado un abreboca de cómo serían los viajes en el tiempo a través de los agujeros de gusano, pero que el futuro no cambia por el principio de auto consistencia de Nóvikov. Así mismo, hemos observado que la paradoja de los abuelos se puede explicar en función de un ejercicio mental desarrollado con lo que sería la auto colisión de una bola de billar, que es capaz de viajar al pasado para poder colisionar consigo misma. Dicho ejercicio es un reflejo clásico de un experimento cuántico realizado por Ringbauer y sus colaboradores, que lograron hacer viajar al pasado a un fotón para que pudiera colisionar consigo mismo.

El corazón de la máquina del tiempo es el agujero de gusano, y como se destacó a lo largo del trabajo, los agujeros de gusano son inestables y su vida útil sería extremadamente corta, por lo que se necesitan mayores desarrollos teóricos para poder permitir los viajes en el espacio-tiempo. Es necesario entonces desarrollar una teoría cuántica de la gravedad para deducir que esos estudios pueden ser realistas. Más aún, la ventana abierta por Greenleaf con los estudios en microondas en un laboratorio, quizás puedan dar luz a la generación de agujeros de gusano, y finalmente ser capaces de corroborar su uso en la travesía temporal.

\section{A. Apéndice A.1}

Karl Schwarzschild (1873-1916) en 1916 [20] describió el campo generado por una estrella esférica de masa $m$ deducida, donde el elemento de línea en coordenadas esféricas está dado por la siguiente ecuación:

$$
\begin{aligned}
d s^{2}=-\left(1-\frac{2 m}{r}\right) d t^{2} & +\left(1-\frac{2 m}{r}\right)^{-1} d r^{2}+ \\
& +r^{2}\left(d \theta^{2}+\operatorname{sen}^{2} \theta d \phi^{2}\right)
\end{aligned}
$$

la cual fue corroborada de manera independiente por Johannes Droste, donde se ha adoptado el sistema de unidades $G=1$ y $c=1$.

Precisamente sobre esta ecuación, Einstein y Rosen publican su trabajo con la esperanza de poder evitar la singularidad cuando $r=0$ y $r=2 m$; para eso, ellos definen una variable $y^{2}=r-2 m$, de modo que al despejar el valor de $r$ e introducirla en la ecuación 35 obtiene la expresión

$$
\begin{aligned}
d s^{2}=- & \frac{y^{2}}{y^{2}+2 m} d t^{2}+4\left(y^{2}+2 m\right) d y^{2}+ \\
& +\left(y^{2}+2 m\right)^{2}\left(d \theta^{2}+\operatorname{sen}^{2} \theta d \phi^{2}\right)
\end{aligned}
$$

donde $y \in(-\infty,+\infty)$, y el puente está justo cuando $y=0$. Es importante señalar que la simplificación de Einstein-Rosen describe la métrica de Schwarzschild y dicho cambio de variable posee un horizonte de sucesos, por lo que es imposible viajar en ambas direcciones.

Si reducimos el espacio a dos dimensiones tal que las coordenadas angulares sean constante, es decir $\theta=\theta_{0} \mathrm{y}$ $\phi=\phi_{0}$, entonces el elemento de línea será igual a:

$$
d s^{2}=-\frac{y^{2}}{y^{2}+2 m} d t^{2}+4\left(y^{2}+2 m\right) d y^{2}
$$

Se determina la geodésica nula en dos dimensiones cuando consideramos $d s=0$, es decir:

$$
\frac{y^{2}}{y^{2}+2 m}\left(\frac{d t^{2}}{d \lambda^{2}}\right)^{2}=4\left(y^{2}+2 m\right)\left(\frac{d y^{2}}{d \lambda^{2}}\right)^{2}
$$

Reagrupando los términos, llegamos a la siguiente expresión: 


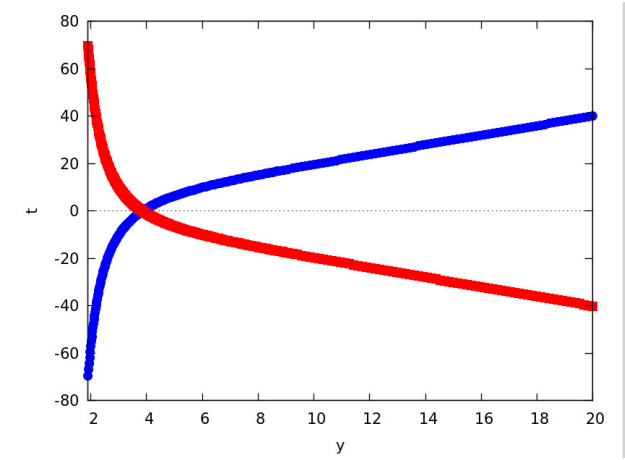

Figura 8: Tiempo radial derivado de las ecuaciones de Einstein-Rosen y masa igual a 20.

$$
\frac{d y}{d t}= \pm \frac{2\left(y^{2}+2 m\right)}{y}
$$

Integrando esta última expresión:

$$
t=t_{0}+\left[2 \sqrt{y^{2}+2 m}-\sqrt{8 m} a \operatorname{senh}\left(\frac{\sqrt{2 n}}{|y|}\right)\right]
$$

Que es un tiempo radial en función de la masa. Visualicemos dicho resultado con ayuda de la figura 8 donde hemos considerado un valor arbitrario de masa $m$ ) igual a 20. La línea azul representa los valores cuando la función es derivada con el signo positivo, mientras que la roja es con el signo negativo. Justo el punto donde se interceptan las dos figuras, delimita el radio de la garganta del agujero de gusano.

\section{B. ApÉNDICE A.2}

Las coordenadas cartesianas que describen el espaciotiempo cuatridimensional están dadas por la siguiente expresión del elemento de línea $d s^{2}$

$$
d s^{2}=-d t^{2}+d x^{2}+d y^{2}+d z^{2}
$$

donde se ha adoptado el sistema de unidades $c=1$. El sistema de coordenadas por excelencia es el esférico en este tipo de problemas, por lo que debemos tener presente cómo se transforma de coordenadas cartesianas a esféricas, de acuerdo a las siguientes relaciones:

$$
\begin{array}{r}
x=r \operatorname{sen}(\theta) \cos (\phi) \\
y=r \operatorname{sen}(\theta) \operatorname{sen}(\phi) \\
z=r \cos (\theta)
\end{array}
$$

De manera que al derivarlas, y reagrupando términos, obtenemos:

$$
\begin{array}{r}
d x=\operatorname{sen}(\theta) \cos (\phi) d r+ \\
\quad r \cos (\phi) \cos (\theta) d \theta- \\
-r \operatorname{sen}(\theta) \operatorname{sen}(\phi) d \phi \\
d y=\operatorname{sen}(\theta) \operatorname{sen}(\phi) d r+r \operatorname{sen}(\phi) \cos (\theta) d \theta+ \\
+r \operatorname{sen}(\theta) \operatorname{sen}(\phi) d \phi \\
d z=\cos (\theta) d r-r \operatorname{sen}(\theta) d \phi
\end{array}
$$

De manera que al elevar al cuadrado, y sustituir las expresiones anteriores en la ecuación 35 , después de reagrupar términos, conseguimos:

$$
d s^{2}=-d t^{2}+d r^{2}+r^{2} d \theta^{2}+r^{2} \operatorname{sen}^{2}(\theta) d \phi^{2}
$$

que corresponde a la expresión de línea en coordenadas esféricas.

Esta última expresión suele simplificarse si empleamos el tensor métrico, donde las coordenadas se describen de la siguiente manera, de modo que la expresión del elemento de línea es de la forma:

$$
d s^{2}=g_{\alpha \beta}(x) d x^{\alpha} d x^{\beta}
$$

donde hemos definido $g_{\alpha \beta}$ como el tensor métrico, el cual se puede representar simplemente como $g_{\alpha \beta}=$ $\operatorname{diag}\left[-1,1, r^{2}, r^{2} \operatorname{sen}^{2}(\theta)\right]$.

\section{Agradecimientos}

El presente trabajo está dedicado a la paciencia de mis padres Raúl 'Viejo' Isea y Blanca 'Mielita' Hernández.

\section{REFERENCIAS}

[1] I.D. Novikov, Phys Rev D Part Fields 45 (1992) 1989

[2] M. Ringbauer, M.A. Broome, C.R. Myers, A.G. White, T.C. Ralph, Nat Commun. 19 (2014) 4145

[3] J. Dolansky y P. Krtous, Phys. Rev. D 82 (2010) 124056

[4] S.W. Hawking, Phys. Rev. D 46 (1992) 603

[5] A. Einstein y N. Rosen, Phys. Rev. 48 (1935) 73

[6] J. Wheeler J. Ann. Phys. 2 (1957) 604

[7] J.A. Khalili, Black Holes, Wormholes \& Time machines, IOP Publishing Ltd, 1999

[8] K. Thorne, The Science of Interstellar, W. W. Norton \& Company, 2014

[9] M. Kaku, Physics of the Impossible, Doubleday Publishing, 2008

[10] L. M. Butcher, Phys. Rev. D 90 (2014) 024019

[11] T. Lafleur, arXiv:1411.5359

[12] M.S. Morris y K.S. Thorne, Amer. J. Phys. 56 (1988) 395

[13] R.W. Fuller y J.A. Wheeler, Phys. Rev. 128 (1962) 919

[14] M. Visser M, Lorentzian Wormholes: From Einstein to Hawking (AIP Series in Computational and Applied Mathematical Physics). AIP Press, New York, Springer. ISBN 1563966530 
[15] O. James, E. Tunzelmann, F. Paul y K.S. Thorne, Am. J. Phys. 83 (2015) 486

[16] C. W. Misner, K.S. Thorne, J.A. Wheeler, Gravitation. San Francisco: W. H. Freeman. ISBN 978-07167-0344-0

[17] S. Carroll, Spacetime and Geometry - An Introduction to General Relativity. pp. 151-159. ISBN 0-8053-8732-3
[18] D. Schurig, J.J. Mock, B.J. Justice, S.A. Cummer, J.B. Pendry, A.F. Starr, D.R. Smith, Science 314 (2006) 977

[19] A. Greenleaf, Y. Kurylev, M. Lassas , y G. Uhlmann, Phys. Rev. Lett. 99 (2007) 183901

[20] K. Schwarzschild, arXiv:physics/9905030 (Traducido por S. Antoci y A. Loinger) 